\title{
Newel-pseudotopologiese vektorruimtes
}

\author{
M.A. Muller \\ Departement Wiskunde, Universiteit van Stellenbosch, Stellenbosch, 7600
}

Ontvang 4 Augustus 1998; aanvaar I6 Februarie 1999

\section{UITTREKSEL}

Pseudotopologiese ruimtes (dit wil sê limietruimtes) is in 1959 deur Fischer ${ }^{1}$ gedefinieer. In hierdie artikel word die teorie van newelpseudotopologiese ruimtes na vektorruimtes uitgebrei. Die begrip begrensdheid word vir newel-pseudotopologiese vektorruimtes ingevoer.

\section{ABSTRACT \\ Fuzzy Pseudo-topological Vector Spaces}

Pseudo-topological spaces (i.e. limit spaces) were defined by Fischer in 1959. In this paper the theory of fuzzy pseudo-topological spaces is applied to vector spaces. We introduce the concept of boundedness in fuzzy pseudo-topological vector spaces.

\section{INLEIDING}

Gestel $X$ is ' $n$ versameling, $I=[0,1]$ en $I^{X}$ is dic versameling van alle funksies van $X$ na $I$. 'n Newclversameling $A$ in $X$ word deur ' $n$ lidmaatskapsfunksic $\mu_{A} \in I^{X}$ gekaraktcriscer. Dic lidmaatskapsfunksic van 'n gewone declversameling van $X$ is sy karakleristieke lunksic. Laal $\underline{\alpha}$ dic newelversameling aandui waarvan die lidmaatskapsfunksic dic konstante funksic met waarde $\alpha$ is. 'n Newelpunt $p$ in $X$ is 'n newelversameling met lidmaatskapslunksic $\mu_{p}$ wat sodanig is dat

$$
\mu_{p}(x)=\left\{\begin{array}{l}
\lambda_{p} \text { as } x=x_{p} \\
0 \text { as } x \neq x_{p},
\end{array}\right.
$$

waar $0<\lambda_{p}<1$. Dic newclpunt $p=\left(x_{p}, \lambda_{p}\right)$ het onderstcuning $x_{p}$ cn waarde $\lambda_{p}$. 'n Newelpunt $p$ behoort $\operatorname{tot}^{7}$ 'n newelversameling $A$ in $X$ (gcskryf $p \in A$ ) as $\mu_{p}\left(x_{p}\right)<\mu_{A}\left(x_{p}\right)$. 'n Gewone punt $y$ word met sy karakteristicke funksic geiddentifisecr. Verder behoort $y$ tot $\Lambda$ as $\mu_{\Lambda}(y)=1$. Met punte word in hierdic artikel beide gewone en newelpunte bedocl, en met versamelings word beide gewone en newelversamelings bedocl.

'n Klas $T \subset l^{X}$ is 'n newcltopologic op $X$ as

(1) $\underline{\alpha} \in \tau^{\circ}$ vir alle $\alpha$

(2) $A, B \in T \Rightarrow A \cap B \in T$,

(3) $A_{j} \in \mathcal{T}$ vir allc $j \in J \Rightarrow \cup\left\{A_{j}: j \in J\right\} \in \mathcal{T}$.

Laat $\mathbf{K}$ dic versameling van skalare ( $\mathbf{R}$ of $\mathbf{C}$ ) met dic gebruiklike topologic $T_{K}$ aandui. Gestel $\tau$ is dic versameling van alle lacrsemikontinuc funksics van $\left(\mathbf{K}, \mathbf{T}_{\mathbf{K}}\right)$ in $I$, dit wil sĉ $\tau=C\left(\left(\mathbf{K}, \mathrm{T}_{\mathbf{K}}\right)\right.$, $I_{r}$ ), waar $I_{r}$ dic versameling $I$ mel dic topologic $\{[\alpha, 1]: \alpha \in I\} \cup$ $\{I\}$ is. Dan is $\tau$ 'n newcltopologic en $(\mathbf{K}, \tau)$ is 'n newcltopologiese ruimtc.

$\mathrm{Na}$ aanleiding van dic idec van 'n filter in 'n verband ${ }^{2}$ word dic volgende delinisic ingevoer: ' $n$ nic-lece klas $\mathcal{F} \subset I^{X}$ word 'n prelilter ${ }^{3}$ genoem as

(1) $A, B \in \mathcal{F} \Rightarrow A \cap B \in \mathcal{F}$,

(2) $A \in \mathcal{F}$ en $A \subset B \Rightarrow B \in \mathcal{F}$,

(3) $\underline{0} \notin \mathscr{T}$.

' $n$ Nic-icë klas $B$ van declversamelings van $X$ word 'n basis van ' $n$ prefilter genoem as

(1) $A, B \in \mathbb{B} \Rightarrow A \cap B \supset C$ vir' 'n sckere $C \in \mathcal{B}$,

(2) $\underline{0} \notin B$. 'n Prefilter $\mathcal{F}$ word deur $\mathcal{B}$ voortgebring as $\mathcal{J}=[\mathcal{B}]=\left\{A \in I^{X}: A\right.$ $\supset B$ vir 'n sekere $B \in \mathscr{B}\}$

1.1 DEFINISIE ${ }^{3}$ As daar vir elke punt $p=(x, \lambda)$ in $X$ 'n nie-le ë versameling $\Delta p$ van prefilters in $X$ bestaan waarvoor

(1) $\mathcal{F} \in \Delta p \Rightarrow \alpha \in \mathscr{F}$ vir alle $\alpha>\lambda$,

(2) $|p|=\left\{\left.A \in\right|^{X}: p \in A\right\} \in \Delta p$,

(3) $\mathscr{F} \in \Delta p$ en $\mathcal{F} \subset \mathcal{G} \Rightarrow \mathcal{G} \in \Delta p$,

(4) $\mathcal{F}, \mathcal{G} \in \Delta p \Rightarrow \mathcal{F} \cap \mathcal{G} \in \Delta p$,

dan word $(X, \Delta)$ ' $n$ newel-pseudotopologiese ruimte genoem. As $\mathcal{I}$ $\in \Delta p$ sê ons dat $\mathcal{F}$ na $p$ konvergeer, ook geskryf $\mathcal{T} \rightarrow p$. (As $p$ 'n gewone punt is, is (l) nie van toepassing nie.)

In hicrdic artikel word aanvaar dat basiese cienskappe van pseudotopologiese ruimtes ${ }^{1,4}$ asook dic notasic wat algemeen in dic tcoric van pscudotopologiese ruimtes gebruik word, bekend is.

Gestcl $(X, \tau)$ is 'n pscudotopologiese ruimte en $p=(x, \lambda)$ is ' $n$ punt in $X$. Vir elke $\mathcal{F} \in \tau x$ laat $G^{\lambda}$ dic oorecnkomstige prefilter wees wat deur dic basis $\left\{A \in I^{X}: \mu_{A}(x)>\lambda, \mu_{A}(\mathscr{H}) \rightarrow \mu_{A}(x)\right.$ in $\left.I_{r}\right\}$ voortgebring word. Die geassosicerde newel-pscudotopologiese strukluur $\Delta_{\tau}$ op $X$ word deur $\Delta_{\tau} p=\{\mathscr{H}: \mathcal{H}$ is 'n prefilter in $X, \mathscr{H} \supset$ $G\}$ vir 'n sckere $\mathcal{F} \in \tau X\}$ gedefinicer. In dic besonder, as $\tau_{\mathbf{K}}$ dic gcbruiklike pscudotopologicse struktuur op $\mathbf{K}$ is (dit wil sĉ, vir $x$ $\epsilon \mathbf{K}$ is $\tau_{\mathbf{K}} x$ dic klas van alle filters in $\mathbf{K}$ wat fyncr is as dic omgcwingsfilter van $x$ met betrekking tot dic gebruiklike topologic $T_{\mathrm{K}}$ op K) word soos hicrbo dic geassosicerde newel-pscudotopologiese struktuur $\Delta_{\tau_{K}}$ verkry, ook genoem dic gebruiklike newclpscudotopologiese struktuur op $K$. In plaas van $\Delta_{\tau_{K}}$ word gerieflikheidshalwe $\Delta_{\mathbf{K}}$ geskryf.

Gestcl $\left(X_{i}, \Delta_{i}\right)$ is 'n newel-pscudotopologicse ruimte vir clke $i$ $=1,2, \ldots, n$. Laat $X=X_{1} \times \ldots \times X_{n}$ en gestel $p_{i}$ is 'n punt in $X_{i}$. Dan word dic newel-pscudotopologiese ruimtc $(X, \Delta)$, waar $\Delta$ dcur $\Delta p$ $=\Delta\left(p_{1}, \ldots, p_{n}\right)=\left\{\mathcal{F}: \mathcal{F}\right.$ is 'n prefilter in $X$ met $\mathcal{F} \supset \mathcal{F}_{1} \times \ldots \times \mathcal{F}_{n}, \mathscr{F}_{i}$ $\in \Delta p_{i}$ vir elke $\left.i\right\}$ gedefinicer word, 'n newclproduk van $\left(X_{1}, \Delta_{1}\right)$, $\ldots,\left(X_{n}, \Delta_{n}\right)$ genoem. Ons herinner dat $\mathcal{T}_{1} \times \ldots \times \mathcal{T}_{n}=\left\{A \in I^{X}: A\right.$ $\supset A_{1} \times \ldots \times A_{n}, A_{i} \in I^{X_{i}}$ waar $A_{i} \supset B_{i}$ vir 'n sckerc $B_{i} \in \mathcal{F}_{i}$, vir clke $i\}$ en dat $A_{1} \times \ldots \times A_{n}$ 'n newelversameling met lidmaatskapsfunksic $\mu$ is, waar $\mu\left(x_{1}, \ldots, x_{n}\right)=\mu_{\Lambda_{1}}\left(x_{1}\right) \wedge \ldots \wedge \mu_{A_{n}}\left(x_{n}\right)$.

\section{NEWEL-PSEUDOTOPOLOGIESE VEKTORRUIMTTS}

Gestel $\left(X, \Delta_{1}\right)$ en $\left(Y, \Delta_{2}\right)$ is newel-pscudotopologicse ruimtes. 'n Afbcelding $f: X \rightarrow Y$ is newelkontinu (mecr presics, $\Delta_{1}-\Delta_{2}$ - 
newelkontinu) by 'n punt $p$ in $X$ as vir enige $\mathcal{F} \in \Delta_{1} p$ dic prefilter $f(\mathcal{F})=\left\{\{f(A): A \in \mathcal{F}\} \mid \in \Delta_{2} f(p)\right.$. Dic afbeelding $f$ is newelkontinu as dit by elke punt van $X$ newelkontinu is.

2.1 STELLING. Gestel $\left(X_{i}, \tau_{i}\right)$ is 'n pseudotopologiese ruimte $(i=$ $1,2,3)$ en $X=X_{1} \times X_{2}$. Laat $\tau$ die produk pseudotopologiese struktuur van $\tau_{1}$ en $\tau_{2}$ op $X$ wees en gestel dat die afbeelding $f$ : $(X, \tau) \rightarrow\left(X_{3}, \tau_{3}\right)$ kontinu is. As $\Delta$ die produk in $X$ van die geassosieerde newel-pseudotopologiese strukture $\Delta_{\tau_{1}}$ en $\Delta_{\tau_{2}}$ is, dan is $f:(X, \Delta) \rightarrow\left(X_{3}, \Delta_{\tau_{3}}\right)$ newel-kontinu.

Bcwys. Gestel $p=\left(p_{1}, p_{2}\right)$ is 'n punt in $X_{1} \times X_{2} \operatorname{met} p_{i}=\left(x_{i}, \lambda_{i}\right)$ in $X_{i}$. Dan $f(p)=\left(f\left(x_{1}, x_{2}\right), \lambda\right)$ met $\lambda=\lambda_{1} \wedge \lambda_{2}$. Gestcl $\mathcal{F} \in \Delta p$, dan $\mathcal{F}$ $\supset \mathscr{F}_{1} \times \mathcal{F}_{2}$ met $\mathscr{F}_{i} \in \Delta p_{i}$ en $\mathcal{F}_{i} \supset \mathcal{G}_{\mathcal{F}_{i}}^{\lambda_{i}}$ vir 'n sekere $\mathcal{H}_{i} \in \tau x_{i},(i=1,2)$. Laat $\mathcal{B} \in \mathcal{G}_{\mathcal{f}\left(g_{1} \times y_{2}\right)}^{\lambda}$, dan volg ${ }^{5}$ dat $\mu_{\mathrm{B}} \circ f \in \mathcal{G}_{\mathrm{H}_{1} \times \mathrm{H}_{2}}^{\lambda}$ en $f\left(\mu_{\mathrm{B}} \circ f\right) \leq \mu_{\mathrm{B}}$. Dus $f(\mathscr{I}) \in \Delta f(p)$, gevolglik is $f$ newelkontinu.

2.2 AFLEIDING. Laat $(E, \tau)$ 'n pseudotopologiese vektorruimte oor $\boldsymbol{K}$ wees. As $E$ van die geassosieerde newel-pseudotopologiese struktuur $\Delta_{\tau}$ voorsien word, $K$ van die gebruiklike newel-pseudotopologiese struktuur $\Delta_{K}$, en $E \times E$ en $K \times E$ van die ooreenkomstige produk newel-pseudotopologiese strukture voorsien word, dan is die afbeeldings $E \times E \rightarrow E((x, y) \rightarrow x+y)$ en $K \times E \rightarrow E$ $((\lambda, x) \rightarrow \lambda x)$ newelkontinu.

2.3 DEFINISIE. ' $n$ Newel-pseudotopologiese vektorruimte is ' vektorruimte $E$ wat van 'n newel-pseudotopologiese ruimtestruktuur $\Delta$ voorsien word en wat sodanig is dat die afbeeldings $E \times E$ $\rightarrow E((x, y) \rightarrow x+y)$ en $K \times E \rightarrow E((\lambda, x) \rightarrow \lambda x)$ newelkontinu is wanneer $\boldsymbol{K}$ die gebruiklike newel-pseudotopologiese struktuur $\Delta_{K}$ het, en $E \times E$ en $K \times E$ van die ooreenkomstige produk newelpseudotopologiese strukture voorsien word, met ander woorde, $\Delta p+\Delta q \subset \Delta(p+q)$ en $\left(\Delta_{\mathbf{K}} \lambda\right)(\Delta p) \subset \Delta(\lambda p)$ vir alle punte $p, q \in E$ en $\lambda \in K$.

2.4 STELLING. Gestel $(E, \Delta)$ is 'n newel-pseudotopologiese vektorruimte en $\alpha$ en y is gewone punte in $K$ en E onderskeidelik. Dan is die afbeelding $f(x)=\alpha x$ en $g(x)=x+y$ van $E$ na $E$ newelkontinu. As $\alpha \neq 0$, is $f^{-1}$ newelkontinu.

Bewys. 'n Afbeclding $k$ van 'n newel-pscudotopologiese ruimte $Y$ na 'n produk $X=\prod_{j \in J} X_{j}$ van newel-pscudotopologiese ruimtes is newelkontinu as en slegs as elke $\pi_{i} \circ k: Y \rightarrow X_{i}$ newelkontinu is, waar $\pi_{i}$ dic kanonicse projeksie ${ }^{3}$ van $X$ in $X_{i}$ is. Aangesien die afbecldings $h_{1}: E \rightarrow \mathbf{K}$ waar $h_{1}(x)=\alpha$ en $h_{2}: E \rightarrow E$ met $h_{2}(x)=$ $x$ ncwelkontinu is, volg dat dic afbeclding $h: E \rightarrow \mathbf{K} \times E$ waar $h(x)$ $=(\alpha, x)$ newelkontinu is. Verder is dic afbeclding $k: \mathbf{K} \times E \rightarrow E$ waar $k(\lambda, x)=\lambda x$ newclkontinu. Dus is $f=k \circ h$ newclkontinu. Op soortgelyke wyse volg dat $g$ newelkontinu is. As $\alpha \neq 0$ volg dic ncwclkontinuïtcit van $f^{-1}$ van die feit dat $f^{-1}(x)=\alpha^{-1} x$.

Gestel $(E, \Delta)$ is ' $n$ newel-pscudotopologicse vektorruimtc. As $F$ 'n declversameling van $E$ en $\alpha$ 'n gewone punt van $\mathbf{K}$ is, laat $\alpha F$ dic becld van $F$ onder die afbeclding $f(x)=\alpha x$ aandui. As $\alpha \neq 0$, volg

$$
\mu_{\alpha \cdot}(t)=\mu_{F}\left(\alpha^{-1} t\right) \text { vir clke } t \in E,
$$

en as $\alpha=0$,

$$
\mu_{\alpha / \cdot}(t)= \begin{cases}\sup \left\{\mu_{F}(y): y \in E\right\} & \text { as } t=0 \\ 0 & \text { as } t \neq 0\end{cases}
$$

As $B$ 'n declversameling van $\mathbf{K}$ is, en $F$ 'n declversameling van $E$ is, laat $B F$ dic beeld van $B \times F$ onder dic afbeclding $g(\lambda, x)=\lambda x$ aandui. Dan

$$
\mu_{B F}(t)= \begin{cases}\sup \left\{\mu_{B \times H}(\alpha, y): \alpha y=t\right\} & \text { as } g^{-1}(t) \neq \emptyset \\ 0 & \text { as } g^{-1}(t)=\emptyset\end{cases}
$$

Gestel $\mathcal{G}$ en $\mathcal{F}$ is prefilters in $\mathbf{K}$ en $E$ onderskeidelik, $\alpha \in \mathbf{K}$ en $x$ $\in E$. Dan is $\alpha \mathcal{F}, \mathcal{G F}$ en $\mathcal{G} x$ prefilters in $E$ wat onderskcidelik deur dic klasse $\{\alpha F: F \in \mathcal{F}\},\{G F: G \in \mathcal{G}, F \in \mathcal{F}\}$ en $\{G x: G \in \mathcal{G}\}$ van deelversamelings van $E$ voortgebring word.

2.5 STELLING. Gestel $U$ is 'n deelversameling van ' $n$ vektorruimte $E$ en $p \in E$. Dan $U-p=V$ as en slegs as $U=p+V$.

Bewys. Aanvaar $U-p=V$. Definicer funksies $f$ en $g$ deur $f(t)=$ $t-p$ en $g(t)=p+t$. Aangesien $V=f(U)$ (Katsaras, Liu 6$)$ volg

$$
\mu_{\bigvee}(r)=\sup \left\{\mu_{U}(s): s \in f^{-1}(r)\right\}=\sup \left\{\mu_{U}(s): s=p+r\right\}
$$

Aangesien $g(V)=p+V$ volg

$$
\mu_{g(V)}(t)=\sup \left\{\mu_{\bigvee}(r): r \in g^{-1}(t)\right\}=\sup \left\{\mu_{\bigvee}(r): r=t-p\right\}=\mu_{U}(t) \text {. }
$$

Derhalwe $U=g(V)=p+V$. Die omgekeerde word soortgelyk bewys.

2.6 STELLING. Gestel $(E, \Delta)$ is 'n newel-pseudotopologiese vektorruimte en $p$ is 'n punt in E. Dan

(1) $\alpha \Delta p \subset \Delta(\alpha p)$ vir elke punt $\alpha$ in $\mathbf{K}$,

(2) $G p \in \Delta(\alpha p)$ vir elke $\mathcal{G} \in \Delta_{\mathrm{K}} \alpha$,

(3) $\Delta p=p+\Delta(p-p)$.

Bewys. (1) Gestel $\mathcal{F} \in \Delta p$. Aangesien $\{\alpha\} \in[\alpha]$ volg $\alpha F=\{\alpha\} F$ $\in[\alpha] \mathcal{F}$ vir elke $F \in \mathcal{F}$. Dus $\alpha \mathcal{F} \subset[\alpha] \mathcal{F}$. Omgekecrd, gestel $A \in$ $[\alpha] \mathcal{F}$. Dan bestaan $B \in[\alpha]$ en $F \in \mathcal{F}$ sodat $A \supset B F \supset \alpha F$. Dus $A$ $\in \alpha \mathcal{F}$ en $[\alpha] \mathcal{F} \subset \alpha \mathcal{F}$. Derhalwe $\alpha \mathcal{F}=[\alpha] \mathcal{F} \in\left(\Delta_{\mathbf{K}} \alpha\right)(\Delta p) \subset \Delta(\alpha p)$. (2) Soos tevore, $G p=G[p] \in\left(\Delta_{\mathrm{K}} \alpha\right)(\Delta p) \subset \Delta(\alpha p)$.

(3) Laat $\mathcal{F} \in \Delta p$ en laat $\mathcal{F}-p$ die prefilter wees wat deur $\{F-p$ : $F \in \mathcal{F}\}$ voortgcbring word. Dan $\mathcal{F}-p=\mathcal{F}+[-p] \in \Delta p+\Delta(-p) \subset$ $\Delta(p-p)$. Dus $\mathcal{F} \in p+\Delta(p-p)$. Omgekcerd, as $\mathscr{H} \in p+\Delta(p-p)$ volg dat $\mathcal{H}=p+\mathcal{F}=[p]+\mathcal{F}$ vir 'n sckere $\mathcal{F} \in \Delta(p-p)$. Dan $p+$ $\mathcal{F} \in \Delta p+\Delta(p-p) \subset \Delta(p+(p-p))=\Delta p$, dus $\mathcal{H} \in \Delta p$.

'n Deelversameling $U$ van 'n newcl-pscudotopologicse ruimtc $(X, \Delta)$ word newel-oop genoem indien vir elke punt $p$ in $U$ 'n oorecnkomstige $V \in \cap\{\mathcal{F}: \mathcal{F} \in \Delta p\}$ bestaan sodat $V \subset U$. As $A$ 'n declversameling van $(X, \Delta)$ is, word die newelsluiting $\vec{A}$ van $A$ gedefinicer as die versameling van alle punte $p$ in $X$ wat sodanig is dat vir ' $n$ sckere $\mathcal{F} \in \Delta p, A \cap F \neq \varnothing$ vir elke $F \in \mathcal{F}$. $A$ word newelgeslote (of $\Delta$-geslote) genoem as $\bar{A}=A$. Dil kan aangetoon word dat 'n declversameling $A$ van $X$ newelgeslote is as en slegs as $X \backslash A$ newel-oop is.

2.7 HULPSTELLING. As A 'n newelgeslote deelversameling van 'n newel-pseudotopologiese vektorruimte $(E, \Delta)$ is, en $\alpha$ en $y$ is gewone punte van $\mathbf{K}$ en $E$ onderskeidelik $(\alpha \neq 0)$ dan is $\alpha A$ en $y+$ A newelgeslote.

Bewys. Gestel $p$ is 'n punt in $\overline{\alpha A}$. Dan bestaan 'n sckere $\mathcal{F} \in \Delta p$ sodat $(\alpha A) \cap F \neq \varnothing$ vir elke $F \in \mathcal{F}$ Laat $\mathcal{G}=\alpha^{-1} \mathcal{F}$ dan $A \cap G \neq$ $\varnothing$ vir elke $G \in G$ en $G \in \alpha^{-1} \Delta p \subset \Delta\left(\alpha^{-1} p\right)$ volgens 2.6. Dan is $\alpha^{-1} p$ 'n punt van $\bar{A}$, dus is $p$ 'n punt van $\alpha A$. Dan volg7 dat $\overline{\alpha A} \subset$ $\alpha A$, dus is $\alpha A$ newelgeslote. Op soortgelyke wyse word aangetoon dat $y+A$ newelgeslote is.

2.8 AFLEIDING. As $U$ 'n newel-oop deelversameling van 'n newel-pseudotopologiese vektorruimte $(E, \Delta)$ is, en $\alpha$ en $y$ is gewone punte van $\mathbf{K}$ en $E$ onderskeidelik $(\alpha \neq 0)$, dan is $\alpha U$ en $y+U$ newel-oop. 
Gestel $(X, \Delta)$ is 'n newel-pscudotopologicsc ruimtc. Dic klas van alle newel-oop declversamelings van $X$ is ' $n$ neweltopologic $\tau_{\Delta}$ op $X$ en word dic neweltopologic genoem wat met die newel-pscudotopologiese struktuur $\Delta$ geassosiecr word. 'n Newelversameling $N$ word 'n omgewing van 'n punt $p$ in $X$ genocm as daar 'n newel-oop versameling $U$ bestaan sodat $p \in U \subset N$. As $p$ 'n punt in $X$ is, laat $\mathcal{N}(p)$ die versameling van alle omgewings van $p$ aandui. Vir elke $p$ is $\mathcal{N}(p)$ 'n prefilter in $X$ en $\mathcal{N}(p) \subset \cap\{\mathcal{F}: \mathcal{F} \in \Delta p\}$.

2.9 AFLEIDING. As $N$ 'n omgewing van ' $n$ punt $p$ in ' $n$ newelpseudotopologiese vektorruimte $(E, \Delta)$ is en $\alpha$ en $y$ is gewone punte in $\mathbf{K}$ en $E$ onderskeidelik $(\alpha \neq 0)$ dan is $\alpha N$ en $y+N$ omgewings van $\alpha p$ en $y+p$ onderskeidelik.

Die bewys van dic volgende benodig ' $n$ bekende resultaat $8,86 \mathrm{cn}$ is soortgelyk aan dic oorcenkomstige resultaat vir pscudotopologicsc ruimtes. 1,4

2.10 STELLING. Gestel $(X, \Delta)$ is 'n newel-pseudotopologiese ruimte wat die volgende eienskappe het:

(I) $\cap\{\mathcal{F}: \mathcal{F} \in \Delta p\} \in \Delta p$ vir elke punt $p$ in $X$.

(2) Gegee ' $n$ punt $p$ en $V \in \cap\{\mathcal{F}: \mathcal{F} \in \Delta p\}$ dan bestaan $W \in \cap\{\mathcal{F}$ : $\mathcal{F} \in \Delta p\}$ sodat $V \in \cap\{\mathcal{G}: \mathcal{G} \in \Delta q\}$ vir elke punt $q$ in $W$.

Dan is $\tau_{\Delta}$ die unieke neweltopologie op $X$ wat sodanig is dat vir elke punt $p, \Delta p$ die klas van alle prefilter verfynings van $\mathcal{N}(p)$ is, dit wil sê $\mathcal{N}(p)=\cap\{\mathcal{F}: \mathcal{F} \in \Delta p\}$.

Omgekeerd, as $(X, T)$ 'n neweltopologiese ruimte is, en as vir elke punt $p$ in $X, \mathcal{N}_{1}(p)$ die versameling van alle T-omgewings van $p$ in $X$ aandui, en as $\Delta_{r} p$ die klas van alle prefilter verfynings van $\mathcal{N}_{\sigma}(p)$ is, dan is $\Delta_{T}$ ' $n$ newel-pseudotopologiese struktuur op $X$ en bevredig $\left(X, \Delta_{\eta}\right)$ (1) en (2) hierbo. (In hierdie sin is newel-pseudotopologiese ruimtes wat (1) en (2) bevredig die neweltopologiese ruimtes.)

Daar bestaan newel-pscudotopologiese ruimtes wat nic neweltopologiese ruimtes is nic. ${ }^{3}$

2.11 AFLEIDING. ' $n$ Newel-pseudotopologiese vektorruimte ( $E$, $\Delta)$ is ' $n$ neweltopologiese vektorruimte as en slegs as $\cap\{\mathcal{F}: \mathcal{F} \in$ $\Delta p\} \in \Delta p$ vir elke punt $p$ in $E$.

Dic bewys is soortgelyk aan dic bewys in dic geval van pscudotopologicse vektorruimtes.

2.12 STEILING. Gestel $(E, \Delta)$ is 'n newel-pseudotopologiese vektorruimte. In $\mathbf{K}$ dui 0 enige newelpunt met ondersteuning 0 , of die gewone punt 0 aan. As $\mathcal{N}(0)$ die versameling van alle omgewings van die punt 0 in $\left(\mathbf{K}, \Delta_{\mathbf{K}}\right)$ is, dan

(l) $\Delta 0+\Delta 0 \subset \Delta 0$,

(2) $\alpha \Delta 0 \subset \Delta 0$ vir elke punt $\alpha$ in $\mathbf{K}$,

(3) $\mathcal{N}(0) \Delta 0 \subset \Delta 0$,

(4) $\mathcal{N}(0) p \in \Delta 0$ vir elke punt $p$ in $E$.

Omgekeerd, gestel $E$ is ' $n$ vektorruimte en $\Delta 0$ is 'n nie-le ̈̈ versameling van prefilters in $E$ wat (1) - (4) hierbo bevredig, asook die voorwaardes van 1.1 met betrekking tot $\Delta 0$. Laat $\Delta p=p+\Delta(p$ - p) vir elke punt $p$ in $E$. Dan is $(E, \Delta)$ ' $n$ newel-pseudotopologiese vektorruimte.

Bewys. Gestel $(E, \Delta)$ is 'n newel-pscudotopologiese vektorruimtc. Dan volg (1) en (3) van 2.3, en (2) en (4) volg van 2.6(1) en 2.6(2) onderskeidelik.

Omgekecrd, gestel $p=\left(x_{p}, \lambda\right)$ is 'n punt in $E$ en $\mathcal{F} \in \Delta p$. Laat $0=$ $(0, \lambda)$. Aangesien $\mathcal{F}-p \in \Delta 0$, volg $\underline{\beta} \in \mathcal{F}-p$ vir clke $\beta>\lambda$. Dan

$$
\mu_{p+\beta}(t)=\sup \left\{\mu_{p}(m) \wedge \mu_{\beta}(n): m+n=t\right\}=\lambda \wedge \beta .
$$

As $\beta>\lambda$ volg $p+\underline{\beta}=\underline{\lambda}$, dus $\underline{\lambda} \in \mathcal{F}$. Aangesien $\underline{\lambda} \subset \underline{\alpha}$ vir clkc $\alpha$ $>\lambda$ volg $\alpha \in \mathcal{F}$ vir elke $\alpha>\lambda$. Derhalwe word aan 1.1(1) voldoen. Dic cienskappe 1.1(2) - 1.1(4) is maklik bewysbaar. Verder $\Delta p+$ $\Delta q=p+\Delta 0+q+\Delta 0 \subset p+q+\Delta 0=\Delta(p+q)$. Dic bewys dat $\left(\Delta_{\mathrm{K}} \alpha\right)(\Delta p) \subset \Delta(\alpha p)$ is soortgelyk aan dic oorecnkomslige bewys vir pscudotopologiese vektorruimtes.

Laat $l_{\varepsilon}$ dic karakteristicke funksic van $\{v:|v| \leq \varepsilon\}$ in $\mathbf{K}$ aandui. As $\rho \in I$ laat $\rho_{\varepsilon}$ die newelversameling $\rho .1_{\varepsilon}$ in $\mathbf{K}$ wecs.

2.13 HULPSTELLING. Gestel $\mathcal{X}(0)$ is die versameling van alle omgewings van die punt $0=(0, \delta)$ in $\left(\mathbf{K}, \Delta_{\mathbf{K}}\right)$ wees, en gestel $\mathcal{N}_{\mathrm{I}}(0)$ is die prefilter $\left\{A \in I^{\mathrm{K}}: A \supset \rho_{\varepsilon}\right.$ vir 'n sekere $\rho \in I$ en $\left.\varepsilon>0\right\}$. Dan $\mathcal{N}(0)=\mathcal{N}_{1}(0)$.

Bewys. Gestel $\mathcal{M}(0)$ is dic filter in $\mathbf{K}$ wat deur $\{\{\nu \in \mathbf{K}:|\nu| \leq \boldsymbol{\varepsilon}\}$ : $\varepsilon \geq 0$ \} voortgebring word. Dan is $\mathcal{T}_{\mathbf{K}} 0$ dic klas van alle filters in $\mathbf{K}$ wat fyner as $\mathcal{M}(0)$ is. Van definisic van dic gebruiklike newelpscudotopologiese struktuur $\Delta_{\mathbf{K}}$ op $\mathbf{K}$ volg dat indien $\rho \geq \delta$,

$$
\rho_{\varepsilon} \in G_{M(0)}^{\delta} \subset G_{g}^{\delta}
$$

vir elke $\mathcal{H} \in \mathcal{T}_{\mathbf{K}} 0$, dus is $\rho_{\varepsilon}$ 'n newel-oop versameling in $\mathbf{K}$ en $\rho_{\varepsilon}$ $\in \mathcal{N}(0)$. Dus $\mathcal{N}_{1}(0) \subset \mathcal{N}(0)$.

Omgckecrd, as $A \in \mathcal{N}(0)$ dan $A \in \cap\left\{\mathcal{K}: \mathcal{X} \in \Lambda_{\mathbf{K}}(0\}\right.$. Aangesicn ook $A \in \mathcal{G}_{\mathscr{M}(0)}^{\delta} \operatorname{volg} \mu_{A}(0)>\delta$ cn $\mu_{A}(\mathcal{M}(0)) \rightarrow \mu_{A}(0)$ in $l_{r}$. Nccm $\delta<\xi$ $<\mu_{A}(0)$ dan bestaan $v>0$ sodat $\left.\mu_{A}(\{z:|z| \leq v\}) \subset \mid \xi, 1\right\}$. Kies $\delta<$ $\beta<\xi$ cn $\varepsilon<v$, dan $\mu_{A}(t) \geq \mu_{\beta \varepsilon}(t)$, dit wil sĉ $A \supset \beta_{\varepsilon}$. Dus $A \in \mathcal{N}_{1}(0)$.

2.14 VOORBEELD. Laat $1_{\varepsilon}$ dic karakteristicke funksic van dic interval $[-\varepsilon, \varepsilon]$ in $\mathbf{R}$ wees. As $\alpha \in l$, laat $\alpha_{\varepsilon}$ dic newelversameling $\alpha .1_{\varepsilon}$ in $\mathbf{R}$ wees. Gestel $0=(0, \delta)$. Definicer 'n newel-pscudotopologiese struktuur $\Delta$ soos volg op dic vektorruimte $E(=\mathbf{R})$ : 'n prefilter $\mathcal{F}$ op $E$ is 'n element van $\Delta 0$ as daar $\varepsilon>0$ bestaan sodat $\alpha_{\varepsilon} \in \mathcal{F}$ vir clke $\alpha \geq \delta$. Definicer $\Delta p=p+\Delta(p-p)$ vir clke punt $p$ in $E$. Beskou $E$ as 'n vektorruimte oor $\mathbf{K}(=\mathbf{R})$ en voorsicn dic skalaarliggaam $\mathbf{K}$ met dic gebruiklike newel-pscudotopologicse struktuur $\Delta_{\mathbf{R}}$. Dan is $(E, \Delta)$ 'n newel-pscudotopologiesc vektorruimte, maar nic 'n neweltopologiese vektorruimte nic.

Dic vereistes van 1.1 met betrekking tot $\Delta 0$ kan maklik nagegaan word. Dic ander vereistes van 2.12 word vervolgens ondersock:

(1) As $\mathcal{F}, \mathcal{G} \in \Delta 0$ dan bestaan $\varepsilon_{1}, \varepsilon_{2}>0$ sodat $\alpha_{\varepsilon_{1}} \in \mathcal{F}$ cn $\beta_{\varepsilon_{2}} \in \mathcal{G}$ vir clke $\alpha, \beta \geq \delta$. Laal $\varepsilon=\varepsilon_{1}+\varepsilon_{2}$. Dan

$$
\begin{aligned}
\mu_{\alpha_{\varepsilon}+\beta_{\varepsilon}}(t) & =\sup \left\{\mu_{\alpha_{\varepsilon}}(m) \wedge \mu_{\beta_{\varepsilon}}(n): m+n=t\right\} \\
& = \begin{cases}\gamma & \text { as }|t| \leq 2 \varepsilon \\
0 & \text { andersins, }\end{cases}
\end{aligned}
$$

waar $\gamma=\alpha \wedge \beta$. Dan $\gamma_{2 \varepsilon} \in \mathcal{F}+\mathcal{G}$ vir elke $\gamma \geq \delta$ en derhalwe $\mathcal{T}+\mathcal{G}$ $\in \Delta 0$.

(2) As $\mathcal{F} \in \Delta 0$ dan bestaan $\varepsilon>0$ sodat $\alpha_{\varepsilon} \in \mathcal{F}$ vir clkc $\alpha \geq \delta$. Gestel $\beta$ is 'n gewone punt in $\mathbf{R}$. As $\beta=0$ volg

$$
\begin{aligned}
\mu_{\beta_{\alpha_{\varepsilon}}}(t) & = \begin{cases}\sup \left\{\mu_{\alpha_{\varepsilon}}(y): y \in \mathbf{R}\right\} & \text { as } t=0 \\
0 & \text { as } t \neq 0\end{cases} \\
& = \begin{cases}\alpha & \text { as } t=0 \\
0 & \text { as } t \neq 0 .\end{cases}
\end{aligned}
$$


Aangesien $\beta_{\alpha_{\varepsilon}}$ die newclpunt $(0, \alpha)$ is en $[(0, \alpha)] \in \beta \mathcal{F}$ vir clke $\alpha$ $\geq \delta$ volg $\beta \mathcal{T} \in \Delta 0$. As $\beta \neq 0$ volg

$$
\begin{aligned}
\mu_{\beta_{\alpha_{\varepsilon}}(t)} & =\mu_{\alpha_{\varepsilon}\left(\beta^{-1} t\right)} \\
& = \begin{cases}\alpha & \text { as }|t| \leq \varepsilon|\beta| \\
0 & \text { andersins, }\end{cases}
\end{aligned}
$$

dus $\alpha_{\varepsilon|\beta|}=\beta \alpha_{\varepsilon} \in \beta \mathcal{F}$ vir clkc $\alpha \geq \delta$. Dus $\beta \mathcal{F} \in \Delta 0$. As $\beta$ dic punt $(x, \lambda)$ in $\mathbf{R}$ is, volg

$$
\begin{aligned}
\mu_{\beta_{\alpha_{\varepsilon}}(t)} & =\sup \left\{\mu_{\beta}(v) \wedge \mu_{\alpha_{\varepsilon}}(n): v n=t\right\} \\
& = \begin{cases}\gamma & \text { as }|t| \leq \varepsilon|x| \\
0 & \text { andersins, }\end{cases}
\end{aligned}
$$

met $\gamma=\lambda \wedge \alpha$. Dan $\gamma_{\varepsilon|x|}=\beta \alpha_{\varepsilon} \in \beta \mathcal{F}$ vir clke $\gamma \geq \delta$. Dus $\beta \mathcal{F} \epsilon$ $\Delta 0$.

(3) As $\mathcal{F} \in \Delta 0$ dan bestaan $\varepsilon>0$ sodat $\alpha_{\varepsilon} \in \mathcal{F}$ vir clke $\alpha \geq \delta$. Laat 0 die newelpunt $(0, \zeta)$ in $\mathbf{K}$ wees. As $\beta \geq \zeta$, is $\beta_{\varepsilon}$ 'n ncwel-oop versameling in $\mathbf{K}$. Verder,

$$
\begin{aligned}
\mu_{\beta_{\varepsilon} \alpha_{\varepsilon}}(t) & =\sup \left\{\mu_{\beta_{\varepsilon}}(v) \wedge \mu_{\alpha_{\varepsilon}}(n): v n=t\right\} \\
& = \begin{cases}\gamma & \text { as }|t| \leq \varepsilon^{2} \\
0 & \text { andersins, }\end{cases}
\end{aligned}
$$

waar $\gamma=\beta \wedge \alpha$. Aangesien $\beta_{\varepsilon} \alpha_{\varepsilon}=\gamma_{\varepsilon^{2}}$ vir clkc $\beta \geq \zeta$ cn $\alpha \geq \delta$, volg $\gamma_{\varepsilon^{2}} \in \mathcal{N}(0) \mathcal{F}$ vir clkc $\gamma \geq \delta$. Dus $\mathcal{N}(0) \mathcal{F} \in \Delta 0$.

(4) Laat $p$ die punt $(x, \lambda)$ in $E$ wees en kies $0<\beta<\delta \wedge \lambda$. Wecreens is $\beta_{\varepsilon}$ 'n newel-oop versamcling in $\mathbf{R}$ en

$$
\mu_{\beta_{v} p}(t)= \begin{cases}\gamma & \text { as }|t| \leq \varepsilon|x| \\ 0 & \text { andersins }\end{cases}
$$

waar $\gamma=\min \{\beta, \lambda\}=\beta$. Dus $\beta_{\varepsilon} p=\beta_{\varepsilon|x|} \subset \alpha_{\varepsilon|x|}$ vir clke $\alpha \geq \beta$. Aangesien dan $\alpha_{\text {elxt }} \in \mathcal{N}(0) p$ vir elke $\alpha \geq \delta$ volg $\mathcal{N}(0) p \in \Delta 0$.

Ten slotte word aangetoon dat $(E, \Delta)$ nic 'n neweltopologiese vektorruimte is nic. Gestel $B \in \cap\{\mathcal{F}: \mathcal{F} \in \Delta 0\}$. Vir $\varepsilon>0$ laat $\mathcal{H}_{\varepsilon}=\{A$ $\in I^{X}: A \supset \alpha_{\varepsilon}$ vir clke $\alpha \geq \delta$ ]. Dan volg $B \in \mathcal{H}_{\varepsilon} \in \Delta 0$, derhalwe $B$ $\supset \cup\left\{\alpha_{\varepsilon}: \alpha \geq \delta\right\}=1_{\varepsilon}$. Aangesien dit vir clkc $\varepsilon \geq 0$ geld, volg $B \supset$ $E$, dus $B=E$. Maar $E \in \mathcal{F}$ vir clkc $\mathcal{F} \in \Delta 0$, dus $\cap\{\mathcal{F}: \mathcal{F} \in \Delta 0\}=$ $\{E\}$. Van dic definisic van $\Delta 0$ volg $\cap\{\mathcal{F}: \mathcal{F} \in \Delta 0\} \notin \Delta 0 \mathrm{cn}$ daarom is $(E, \Delta)$ nic 'n neweltopologicse vektorruimte nic.

\section{Begrensdheid}

'n Declversameling $A$ van 'n newcl-pscudotopologiese vektorruimtc $(E, \Delta)$ is gebalanscerd as $\lambda A \subset A$ vir clkc $\lambda \in \mathbf{K}$ waar $|\lambda| \leq$ 1. Dic gebalansecrde omhulsel 6 van $A$ is $\cup\{\lambda A:|\lambda| \leq 1\}$ en dit is die kleinste gebalansecrde declversameling van $E$ wat $A$ bevat.

'n Declversameling $B$ van 'n neweltopologiese vektorruimte word begrens genocm ${ }^{10}$ as vir elke omgewing $U$ van $0 \mathrm{cn}$ clke $\alpha$ $<\beta$ waar $\beta \leq \mu_{U}(0)$ 'n $t>0$ bestaan sodat $t(\alpha \cap B) \subset U$. Gestel $B$ is 'n begrensde declversameling en $U$ is ' $n$ omgewing van 0 in 'n neweltopologiese vektorruimte. Daar bestaan' 'n gebalansecrde omgewing $W$ van 0 met $W \subset U$, dus as $\alpha<\beta$ met $\beta \leq \mu_{W}(0)$, volg $t(\underline{\alpha} \cap B) \subset W$ vir'n sckere $t>0$. Laal $N_{t}=\{\gamma:|\gamma| \leq t\}$, dan $N_{t}(\underline{\alpha}$ $\cap B) \subset W \subset U$. As $\mathcal{X}(B)$ die prefilter aandui wat deur $\{\underline{\alpha} \cap B: \alpha$ $>0\}$ voortgebring word, dan konvergecr $\mathcal{N}(0) \mathcal{X}(B)$ na 0 . Hierdic oorwegings dien as motivering vir die volgende:
3.1 DEFINISIE. (1) Gestel $B$ is 'n deelversameling van 'n newel. pseudotopologiese vektorruimte $(E, \Delta)$. Dan is $B$ begrens (dit wil sê $\Delta$-begrens) as of $B=\emptyset$, of $\mathcal{N}(0) \mathcal{X}(B) \in \Delta(0$ wanneer $B \neq \emptyset$.

(2) ' $n$ Afbeelding van die newel-pseudotopologiese vektorruimte $(E, \Delta)$ na die newel-pseudotopologiese vektorruimte $\left(E_{1}, \Delta_{1}\right)$ is begrens as dit begrensde deelversamelings van $E$ op begrensde deelversamelings van $E_{1}$ afbeeld.

3.2 HULPSTELLING. ' $n$ Nie-leë deelversameling $B$ van 'n newelpseudotopologiese vektorruimte $(E, \Delta)$ is begrens as en slegs as $\mathcal{N}(0) B \in \Delta 0$.

Bewys. Aangesien

$$
\begin{aligned}
\mu_{\rho_{\varepsilon}}(\alpha \cap B)(t) & =\sup \left\{\mu_{\rho_{\varepsilon}}(v) \wedge \mu_{(\alpha \cap B}(n): v n=t\right\} \\
& =\sup \left\{\rho \wedge \alpha \wedge \mu_{B}(n): v n=t,|v| \leq \varepsilon\right\} \\
& =\sup \left\{\mu_{(\rho \wedge \alpha)_{\varepsilon}}(v) \wedge \mu_{B}(n): v n=t\right\} \\
& =\mu_{(\rho \wedge \alpha)_{\varepsilon} B}(t)
\end{aligned}
$$

volg $\left.\rho_{\varepsilon}(\alpha \cap B)=\rho \wedge \alpha\right)_{\varepsilon} B$. Dus $\mathcal{N}(0) \not(B) \subset \mathcal{N}(0) B$.

Omgckecrd, $\rho_{\varepsilon} B=\rho_{\varepsilon}(1 \cap B)$, dus $\mathcal{N}(0) B \subset \mathcal{N}(0) \mathcal{X}(B)$.

3.3 HULPSTELLING. Gestel $(E, \Delta)$ is 'n newel-psendotopologiese vektorruimte, $A$ en $B$ is begrensde deelversamelings van $E$ en $\alpha \in \mathbf{K}$.

(l) As $\left(E_{1}, \Delta_{1}\right)$ 'n newel-pseudotopologiese vektorruimte is en $f$ : $E \rightarrow E_{1}$ is ' $n$ newelkontinue lineêre afbeelding, dan is f begrens.

(2) $A \cup B, \alpha A$ cn $A+B$ is begrens.

(3) As $C \subset A$ dan is $C$ begrens.

(4) Elke eindige versameling van punte in $E$ is begrens.

(5) Die gebalanseerde omhulsel van A is begrens.

Bewys. (1) Laat $F \in f(\mathcal{N}(0) A)$, dan $F \supset f\left(\rho_{\mathbb{E}} \mathcal{A}\right)$ vir 'n sckerc newelversameling $\rho_{\varepsilon} \in \mathcal{N}(0)$. Verder

$$
\begin{aligned}
& \mu_{f\left(\rho_{\mathrm{r}} \wedge\right)}(t)=\sup \left\{\mu_{\mathrm{\rho}_{\mathrm{r}} \wedge}(s): s \in f^{-1}(t)\right\} \\
& =\sup \left\{\sup \left\{\mu_{\rho_{\varepsilon}}(v) \wedge \mu_{\Lambda}(n): v n=s\right\}: s \in f^{1}(t)\right\} \\
& =\sup \left\{\sup \left\{\rho \wedge \mu_{\Lambda}(n): v n=s,|\nu| \leq \varepsilon\right\}: s \in f^{\prime}(t)\right\} \\
& =\sup \left\{\sup \rho \wedge \mu_{v A}(s): s \in f^{\prime}(t)\right\} \\
& |v| \leq \varepsilon \\
& =\sup _{|v| \leq \varepsilon} \sup \left\{\rho_{\varepsilon} \wedge \mu_{v A}(s): s \in f^{-1}(t)\right\} \\
& =\sup \left\{\rho \wedge \mu_{(v \mathcal{})}(t):|v| \leq \varepsilon\right\}
\end{aligned}
$$

en

$$
\begin{aligned}
\mu_{\rho_{\mathcal{f}} f(\Lambda)}(t) & =\sup \left\{\mu_{\rho_{\mathrm{\varepsilon}}}(v) \wedge \mu_{f(\Lambda)}(n): v n=t\right\} \\
& =\sup \left\{\rho \wedge \mu_{f(\Lambda)}(n): v n=t,|v| \leq \varepsilon\right\} \\
& =\sup \left\{\rho \wedge \mu_{v f(\Lambda)}(t):|v| \leq \varepsilon\right\} .
\end{aligned}
$$

Maar $f(v A)=v f(A)$ (Katsaras, Liu', Proposition 2.2), dus $f\left(\rho_{\mathcal{A}} \mathcal{A}\right)=$ $\rho_{\mathrm{E}} f(\Lambda)$. Dan $F \in \mathcal{N}((0) f(A)$, gevolglik $f(\mathcal{N}(0) A) \subset \mathcal{N}(0) f(A)$. Aangesien $f(\mathcal{N}(0) A) \in \Delta_{1} 0$ volg dat $\mathcal{N}(0) f(A) \in \Delta_{1} 0$, dil wil sê $f(\Lambda)$ is begrens.

(2) Ons bewys dat $A+B$ begrens is. Laat $F \in \mathcal{N}(0) A+\mathcal{N}(0) B$. Daar bestaan newelversamclings $\lambda_{\varepsilon_{1}}, v_{\varepsilon_{2}} \in \mathcal{N}(0)$ sodat $\lambda_{\varepsilon_{1}} \wedge+v_{\varepsilon_{2}} B$ $\subset F$. Laat $\varepsilon=\varepsilon_{1} \wedge \varepsilon_{2}$ en $\rho=\lambda \wedge \nu$. Ons toon aan dat

$$
\rho_{\varepsilon}(A+B) \subset \rho_{\varepsilon} A+\rho_{\varepsilon} B
$$

want dan sal $\rho_{\varepsilon}(A+B) \subset \lambda_{\varepsilon_{1}} A+v_{\varepsilon_{2}} B \subset F$ cn dus $\mathcal{N}(()) A+\mathcal{N}((0) B$ $\subset \mathcal{N}(0)(A+B)$. Aangesien $\mathcal{N}(0) A+\mathcal{N}(0) B \in \Delta 0+\Delta 0 \subset \Delta 0$ volg $\mathcal{N}(0)(A+B) \in \Delta 0$, dil wil sê $A+B$ is begrens. Nou word (*) bewys: 


$$
\begin{aligned}
\mu_{\rho_{\varepsilon}(\Lambda+B)}(t) & =\sup \left\{\mu_{\rho_{\varepsilon}}(v) \wedge \mu_{\Lambda+B}(n): v n=t\right\} \\
& =\sup \left\{\rho \wedge \mu_{\Lambda+B}(n): v n=t,|v| \leq \varepsilon\right\} \\
& =\sup \left\{\rho \wedge \mu_{v(\Lambda+B)}(t):|v| \leq \varepsilon\right\}
\end{aligned}
$$

Maar

$$
\begin{aligned}
\mu_{\rho_{\varepsilon} A+\rho_{\mathrm{E}} B}(t) & =\sup \left\{\mu_{\mathrm{\rho}_{A} A}(m) \wedge \mu_{\mathrm{\rho}_{\varepsilon} B}(n): m+n=t\right\} \\
& =\sup \left\{\left(\sup _{|r| \leq \varepsilon} \rho \wedge \mu_{\Lambda}\left(\frac{\pi}{r}\right)\right) \wedge\left(\sup _{|s| \leq \varepsilon} \rho \wedge \mu_{B}\left(\frac{m}{r}\right)\right): m+n=t\right\} \\
& \geq \sup \left\{\sup _{|r| \leq \varepsilon} \rho \wedge \mu_{r A}(m) \wedge \mu_{r B}(n): m+n=t\right\} \\
& =\sup \left\{\rho \wedge \sup _{m+n=t} \mu_{r A}(m) \wedge \mu_{r B}(n):|r| \leq \varepsilon\right\} \\
& =\sup \left\{\rho \wedge \mu_{r \Lambda+r l B}(t):|r| \leq \varepsilon\right\} .
\end{aligned}
$$

Verder $r A+r B=r(A+B)$ (Katsaras, Liu' ${ }^{6}$, Corollary 2.3). Dus volg (*). Dic ander bewerings word soorlgelyk bewys.

3.4 DEIJINISIE. 'n Newel-pseudotopologiese vektorruimte $(E, \Delta)$ is lokaalbegrens as elke $F \in \Delta 0$ 'n begrensde deelversameling van E bevat.

3.5 VOORBEELID. Dic newel-pseudotopologiese vektorruimte in 2.14 is lokaalbegrens. Gestcl $0=(0, \delta)$ cn $\mathcal{F} \in \Delta 0$. Van definisic bestaan 'n sckere $\varepsilon>0$ sodat $\alpha_{\varepsilon} \in \mathcal{F}$ vir clkc $\alpha \geq \delta$. Laat $\rho_{\varepsilon_{1}} \alpha_{\varepsilon} \in$ $\mathcal{N}(0) \alpha_{\mathbf{r}}$. Dan volg

$$
\begin{aligned}
\mu_{\rho_{\varepsilon, 1} \alpha_{\varepsilon}}(t) & =\sup \left\{\mu_{\rho_{\mathrm{E}: 1}}(\nu) \wedge \mu_{\alpha_{\varepsilon}}(n): v n=t\right\} \\
& =\sup \left\{\rho \wedge \alpha: v n=t,|v| \leq \varepsilon_{\mathrm{I}},|n| \leq \varepsilon\right\} .
\end{aligned}
$$

Laat $\gamma=\rho \wedge \alpha$ dan volg $\rho_{\varepsilon_{1}} \alpha_{\varepsilon}=\gamma_{\varepsilon \varepsilon_{1}}$ cn $\gamma_{\varepsilon \varepsilon_{1}} \in \mathcal{N}(0) \alpha_{\varepsilon}$ vir clke $\gamma \geq$ $\alpha$. Van definisic van $\Delta 0$ volg $\mathcal{N}(0) \alpha_{\varepsilon} \in \Delta 0$, gevolglik is $\alpha_{\varepsilon}$ begrens.

3.6 DEFINISIE. ' $n$ Newel-pseudotopologiese vektorruimte $(E, \Delta)$ is ewewigtig ("equable") as vir elke $\mathcal{T} \in \Delta 0$ 'n ewewigtige prefilter $\mathcal{G}$ (dit wil sê $\mathcal{N}(0) \mathcal{G}=\mathcal{G}$ ) in $E$ bestaan sodat $\mathcal{G} \in \Delta 0$ cn $\mathcal{G} \subset \mathcal{F}$.

3.7 IIULPSTEL _I ING. Gestel $(E, \Delta)$ en $\left(E_{1}, \Delta_{1}\right)$ is newel-pseudotopologiese vektorruimtes en $f: E \rightarrow E_{1}$ is 'n linêेre afbeelding. $\Lambda s(E, \Delta)$ ewewigtig is en as elke prefilter $\mathcal{F} \in \Delta 0$ ' $n$ deelversameling $M$ van $E$ bevat wat die eienskap het dat $f(M)$ begrens is, dan is $f$ newelkontinu.

Bewys. Gestel $\mathscr{F} \in \Delta 0$. Volgens aanname bestaan 'n cwewigtige prelilter $\mathcal{G} \in \Delta 0$ en $M \in \mathcal{G}$ sodat $\mathcal{F} \supset \mathcal{G}$ en $\mathcal{N}(0) f(M) \in \Delta_{1}(0$. Laat $V \in \mathcal{N}(0) f(M)$, dan bestaan ' $\mathrm{n}$ ncwclversamcling $\rho_{\varepsilon} \in \mathcal{N}(0)$ sodat $V \supset \rho_{\varepsilon} f(M)=f\left(\rho_{\varepsilon} M\right)$. Aangesien $\rho_{\varepsilon} M \in \mathcal{N}(0) M \subset \mathcal{N}(0) G \subset \mathcal{F}$ volg $V \in f(I)$, gevolglik $\mathcal{N}\left((0) f(M) \subset f(g)\right.$. Dus $f(\mathscr{F}) \in \Delta_{1}(0$.

3.8 DEFINISIE. ' $n$ Newel-pseudotopologiese vektorruimte $(E, \Delta)$ is bornologies as vir elke prefilter $g \in \Delta 0$ 'n ooreenkomstige begrensde versameling $B$ bestaan sodat $\mathcal{T} \supset \mathcal{N}(0) B$.

3.9 STELLING. Gestel $(E, \Delta)$ is 'n newel-pseudotopologiese vektorruimte. Beskou die volgende bewerings:

(I) $(E, \Delta)$ is bornologies.

(2) $(E, \Delta)$ is ewewigtig en lokaalbegrens.

(3) Elke begrensde lineêre afbeelding $f: E \rightarrow E_{1}$ (watar $\left(E_{1}, \Delta_{1}\right)$ enige newel-pseudotopologiese vektorruimte is) is newelkontinu. $\operatorname{Ian}(I) \Rightarrow(2) \Rightarrow(3)$.

Bewys. (1) $\Rightarrow(2)$ : As $\mathcal{F} \in \Delta 0$ dan bestaan daar 'n begrensde versamcling $B$ sodat $\mathcal{F} \supset \mathcal{N}(0) B$. Aangesien $\mathcal{N}(0) B$ 'n cwewigtige filter in $E$, is, is $(E, \Delta)$ ewewigtig. I aat $B^{\prime}$ dic gebalansecrde omhulsel van $B$ wees. Dan volg $B^{\prime} \in \mathcal{N}(0) B^{\prime} \subset \mathcal{N}(0) B$, dus $B^{\prime} \in \mathcal{I}$. Volgens 3.3 is $B^{\prime}$ begrens, dus is $(E, \Delta)$ lokaalbegrens.

$(2) \Rightarrow(3)$ : Hicrdic implikasic volg van 3.7 .

\section{SUMMARY}

The theory of pscudo-topological vector spaces (i.c. limit vector spaces) is well-known, and recently the theory of fur:ry pscudotopological spaces was developed by K.C. Min. In this paper furzy pscudo-topological structure is defined on vector spaces. A characterization of fuzry pscudo-topological vector spaces is oblained, and an example is given of a furzy pscudo-topological vector space which is not a furzy topological vector spacc. A concept of boundcdness is introduced for fuzzy pscudo-topological vector spaces. The definition of a bornological fuzzy pscudo-topological vector space leads to the result that a bounded lincar mapping on a bornological fuzzy pscudo-topological vector space is furzy continuous.

\section{LITERATUURVERWYSINGS}

1. Fischcr, H.R. (1959). Limesräumc, Math. Ann., 137, 269 - 303.

2. Bourbaki, N. (1966). General Topology (Hcrmann, Paris).

3. Min, K.C. (1989). Fuzay limit spaccs, Fuzzy Sets and Systems, 32, 343 -357 .

4. Gählcr, W. (1977/8). Grundstrukturen der Analysis I \& // (Birkhäuscr Verlag, Basel).

5. Weiss, M.D. (1975). Fixed points, scparation and induccd topologics for fuzzy sets, J. Math. Anal. Appl., 50, 142 - 150.

6. Katsaras, A.K., Liu, D.B. (1977). Fuzzy vector spaces and fuz:ry topological vector spaces, J. Math. Anal. Appl., 58, 135 - 146.

7. Srivastava, R., Lal, S.N., Srivastava, A.K. (1981). Jur:ry llausdorff topological spaces, J. Math. Anal. Appl., 81, 497 - 506.

8. Kim, C.Y. (1987). Fux:ry topology and ncighbourhood sysicms, I. Nat. Acad. Sci. Korea Nat. Sci., Series XXVI, 1 - 13.

9. Katsaras, A.K. (1981). Fuzzy topological vector spaces I, fuzzy Sets and Systems, 6, $85-95$.

10. Katsaras, A.K. (1984). Furzy topological vector spaces II, Fuzzy Sets and Systems, 12, 143- 154. 